\title{
Numerical Simulation of Flow Control by a Heat Pulse under Aerodynamic Hysteresis in Supersonic Flow over an Axisymmetric Body with Annular Cavity
}

\author{
A.S. Shishaeva ${ }^{1,2}$, M.M. Simonenko ${ }^{1}$, S.V. Guvernyuk ${ }^{1}$, A.A. Aksenov ${ }^{3}$ \\ ${ }^{1}$ Institute of mechanics, Lomonosov Moscow State University, Moscow, 119192, Russia \\ ${ }^{2}$ Tesis LTD, Moscow, 127083, Russia \\ ${ }^{3}$ Joint Institute for High Temperatures of Russian Academy of Sciences (JIHT), \\ Moscow, 125412, Russia \\ anastasiashishaeva@,rambler.ru
}

\begin{abstract}
A numerical simulation of flow control by an artificial heat actuator is presented. Investigation is performed for supersonic flow over an axisymmetric annular cavity under aerodynamic hysteresis conditions. The simulation is carried out using the FlowVision computational software package. The comparison of results of numerical simulation and experimental values is performed. The influence of position, power and width of heat pulse on the gas flow is studied.
\end{abstract}

Keywords: flow control, supersonic flow, annular cavity, hysteresis, numerical simulation. 


\title{
Численное моделирование управления потоком с помощью теплового импульса при сверхзвуковом обтекании осесимметричного тела с каверной в условиях аэродинамического гистерезиса
}

\author{
А.С. Шишаева ${ }^{1,2}$, М.М. Симоненко ${ }^{1}$, С.В. Гувернюк ${ }^{1}$, А.А. Аксенов ${ }^{3}$ \\ ${ }^{1}$ Научно-исследовательский институт механики МГУ, \\ Россия, Москва, 119192, Мичуринский проспект, д. 1 \\ ${ }^{2}$ ООО «ТЕСИС», Россия, Москва, 127083, ул. Юннатов, д. 18 \\ ${ }^{3}$ ОИВТ РАН, Россия, Москва, 125412, ул. Ижорская, д.13, стр.2, \\ anastasiashishaeva@rambler.ru
}

\begin{abstract}
Аннотация
Представлены результаты численного моделирования управления потоком с помощью искусственного теплового источника при сверхзвуковом обтекании осесимметричного тела с кольцевой каверной на режиме гистерезиса. Моделирование выполнено с использованием программного комплекса FlowVision. Исследовано влияние местоположения, мощности и длительности действия теплового источника на перестройку результирующего течения.
\end{abstract}

Ключевые слова: управление потоком, сверхзвуковой поток, кольцевая каверна, гистерезис, численное моделирование.

\section{1. Введение}

Вопросы управления ударно-волновыми и отрывными структурами при сверхзвуковом обтекании тел составляют одно из актуальных направлений исследований в газовой динамике. При этом можно выделить два основных принципа управления. Первый - формирование искусственных возмущений непосредственно в пристеночной области, например - на поверхности обтекаемого тела [1-5]. Второй -воздействие на набегающий сверхзвуковой поток с целью создания в нем областей неоднородности, которые затем влияют на структуру обтекания тела [6-10]. Разнообразие вариантов внесения искусственных возмущений включает механические, струйные и энергетические способы. Вынужденные колебания участка границы обтекаемого тела порождают акустические волны, способные влиять на устойчивость и отрыв пограничного слоя [1]. Системы вдува/отсоса газа с поверхности тел традиционно применяются для изменения параметров пограничного слоя и воздействия на отрывные структуры [2]. Относительно новым является энергетический способ, при котором в пограничный слой на поверхности тела подводится тепло, например, с помощью скользящего поверхностного разряда, что может влиять на ламинарно-турбулентный переход [3] или на конфигурацию ударно-волновых структур и местных сверхзвуковых зон на крыловом профиле в трансзвуковом потоке [4-5]. Воздействие на набегающий сверхзвуковой поток перед затупленным телом можно осуществлять с помощью различных выступающих вперед механических элементов (в виде игл с насадками или без), метаемых вперед твердых частиц, выдува встречных высоконапорных газовых или жидкостных струек. В последнее время наибольшее 
внимание уделяется энергетическому способу воздействия, при котором в набегающем сверхзвуковом потоке создаются локализованные источники тепловыделения. Впервые такой способ управления был предложен в работе [6]. С помощью численного моделирования совместного обтекания постоянного источника внешнего энергоподвода и расположенных вниз по потоку различных тел, показана возможность существенного снижения их волнового сопротивления [6-7]. Возможность практической реализации локализованного энергоподвода в сверхзвуковой поток описана в работе [8]. В этом эксперименте подвод энергии осуществлялся в виде последовательности коротких оптических разрядов большой мощности. Соответствующая теоретическая модель импульсно-периодического энергоподвода, позволяющая варьировать частоту следования импульсов при заданной средней мощности источника, предложена в [9], где впервые была рассчитана зависимость эффективности теплового источника от его частоты. Во многих последующих экспериментальных и расчетных исследованиях различных авторов было также получено, что внесение возмущений в набегающий сверхзвуковой поток перед обтекаемым телом позволяет существенным образом изменять картину обтекания тела и снижать его волновое сопротивление. Однако выполненный в [10] анализ показал, что в большинстве случаев такой подход к снижению сопротивления летящего тела является малоэффективным, поскольку мощность энергоподвода, необходимая для внесения возмущения достаточной интенсивности, превышает мощность, которую удается сэкономить за счет снижения волнового сопротивления. При этом еще в работе [11] было показано, что для формирования в сверхзвуковом потоке динамически эквивалентных неоднородностей с помощью источников тепла или механического импульса (приводящих к одинаковому снижению волнового сопротивления тел), более энергозатратным всегда является подвод тепла. Тем не менее, возможны ситуации, когда задачей управления является не формирование и последующее удержание новых конфигураций течения, а лишь провоцирование перестройки течения из одного из возможных состояний в другое. Пример такой возможности был указан в работе [12]. В подобных случаях потребная мощность источника искусственных возмущений может быть приемлемой.

Известно, что стационарное сверхзвуковое обтекание систем тел и их элементов может обладать свойством неединственности, когда реализующуюся структуру течения определяют не только физические параметры потока и геометрия тела, но и предыстория формирования течения. В частности, на течение могут оказывать влияние начальные условия, характер изменения скорости внешнего потока в процессе установления обтекания тела, скорость и направление движения элементов конструкции тела и прочее. В таких случаях наблюдаются гистерезисные зависимости характеристик обтекания тела, например, аэродинамического сопротивления, от указанных параметров задачи [13-18]. Гистерезисными свойствами обладают многие отрывные течения, простейшим примером является сверхзвуковое обтекание плоских прямоугольных вырезов [19] и кольцевых каверн на осесимметричных телах [20-23]. В настоящее время развиты вычислительные технологии, способные адекватно моделировать гистерезисные явления при сверхзвуковом обтекании каверн [24-27]. Численно воспроизведены наблюдавшиеся в эксперименте [22] гистерезисные зависимости при непрерывном увеличении и уменьшении длины каверны, а также переходные нестационарные явления при переключении режимов обтекания от одной схемы к другой [27].

Сверхзвуковые турбулентные течения около кольцевых каверн представляют большой практический интерес, так как реализуются в областях между выступами и около всевозможных выемок и полостей на корпусах различных летательных аппаратов. В зависимости от отношения длины каверны к ее глубине течение может быть двух видов: с открытым и замкнутым типами взаимодействия [19-23]. Когда отношение длины каверны к глубине велико, реализуется замкнутое течение. В этом случае внешний сверхзвуковой поток заходит в каверну и присоединяется к поверхности дна каверны. При этом, около переднего и заднего уступов каверны формируются две изолированные области отрыва потока. Когда соотноше- 
ние между длиной и глубиной каверны мало, реализуется только открытое течение, при котором всю каверну от передней до задней стенки занимает дозвуковое отрывное течение, отделенное от внешнего потока турбулентным слоем смешения. На определенном интервале соотношений между длиной и глубиной каверны в зависимости от предыстории обтекания может реализовываться как открытый, так и замкнутый режим течения в каверне, т.е. имеет место гистерезис. При этом сопротивление обтекаемого тела с замкнутым режимом течения в каверне может существенно превышать сопротивление аналогичного тела с открытым режимом течения в каверне [21, 23, 27].

Важной практической задачей является поиск способов управления режимами течения в каверне, в частности, способов переключения режимов обтекания каверны, а также способов затягивания перехода от открытой каверны к замкнутой. Известно $[21,23]$, что в области гистерезиса перестройка течения в осесимметричной каверне от замкнутой схемы к открытой схеме происходит при изменении угла атаки необратимым образом. Целью данной работы является определение условий, при которых перестройка замкнутой схемы течения в осесимметричной каверне к открытой схеме происходит под воздействием искусственного теплового возмущения.

\section{2. Постановка задачи численного моделирования}

Рассматривается осесимметричное сверхзвуковое обтекание цилиндрического тела с коническим наконечником и кольцевой каверной прямоугольного сечения (рис. 1). Угол полураствора конуса $\beta=20^{\circ}$, диаметр тела и основания конуса $D=45 \mathrm{Mм}$, длина каверны $L=86.4$ мм, глубина каверны $h=8$ мм. Параметры набегающего потока соответствуют параметрам потока в эксперименте [22] и расчете [27]: $M_{\infty}=3, p_{\infty}=11706$ Па, $T_{\infty}=98.2 \mathrm{~K}$, $V_{\infty}=596.5 \mathrm{~m} / \mathrm{c}, \operatorname{Re}(D)=1.58 \times 10^{6}$. Рабочая среда - воздух. Для таких условий численно определены [27] границы области гистерезиса $(7.8<L / h<11.3)$. Далее в данной работе для исследований используется та же модель, что и в работе [27].

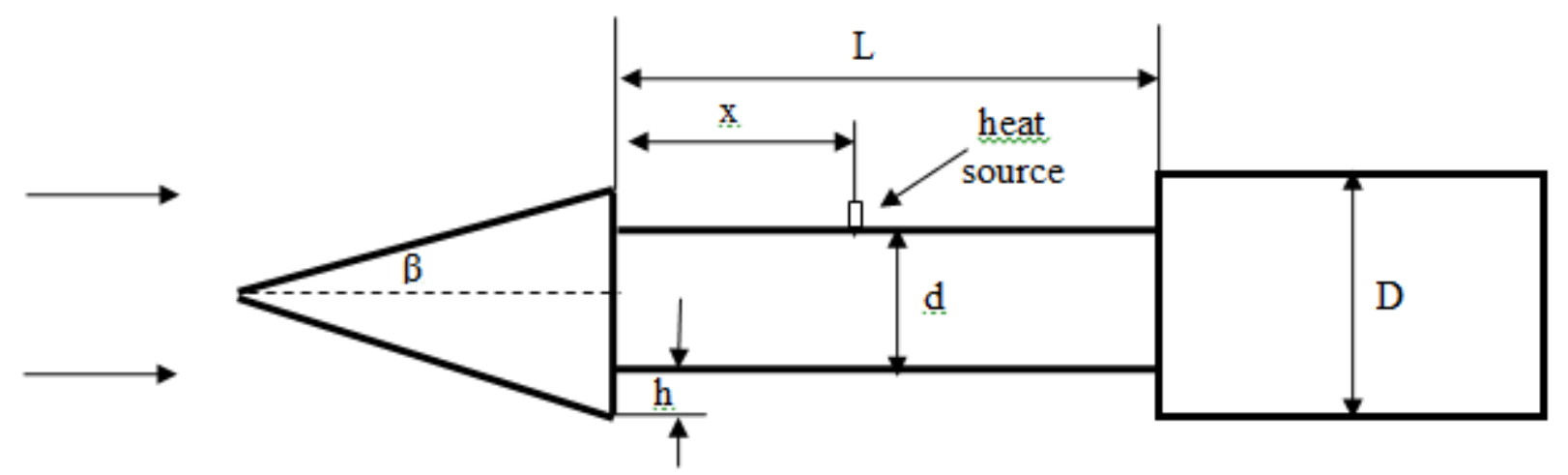

Рис. 1. Геометрия тела с каверной

В рассматриваемом случае $L / h=10.8$, в такой каверне могут реализовываться как замкнутая, так и открытая схема течения. Для исследования влияния возмущения на течение в каверне в области гистерезиса в качестве начального условия используется решение, соответствующее замкнутой схеме течения в каверне [27].Возмущение в решение вносится посредством теплового источника, расположенного на дне каверны (см. рис. 1).Источник представляет собой кольцевую область пространства, в которой в момент времени $t_{d}=0$ на заданный временной интервал $\tau_{d}$ включается тепловыделение заданной мощности $N_{d}$. Размер области тепловыделения равен по ширине 0.9 мм, по высоте -2.7 мм. Основными размерными параметрами, характеризующими задачу, являются: $x_{d}$ - расстояние от переднего уступа каверны до источника (м), $N_{d}$ - мощность тепловыделения в источнике (Вт), $\tau_{d}-$ время работы источника (c). В безразмерном виде 


$$
x=\frac{x_{d}}{h} ; \tau=\frac{V_{\infty} \tau_{d}}{L} ; N=\frac{N_{d}}{\frac{\rho_{\infty} V_{\infty}^{2}}{2} \pi\left(\frac{D}{2}\right)^{2} V_{\infty}}=\frac{8 N_{d}}{\pi \rho_{\infty} V_{\infty}^{3} D^{2}} ; t=\frac{V_{\infty} t_{d}}{L}
$$

Численное моделирование выполняется с помощью вычислительной технологии, реализованной в пакете FlowVision. Решаются нестационарные уравнения Рейнольдса, уравнение энергии, записанное через полную энтальпию, и уравнения стандартной $k-\varepsilon$ модели турбулентности. Для решения уравнений используется неявная схема расщепления второго порядка аппроксимации по пространству и первого порядка по времени. Описание математических моделей, численных методов и граничных условий, используемых в пакете FlowVision при моделировании турбулентных течений, приведено в работах [28-30]. В расчетах используется осесимметричная расчетная область с размерами 0.25 м на 0.125 м, ось которой совпадает с осью обтекаемого тела. Расстояние от входной границы до обтекаемого тела составляет 0.0355 м. Кормовая часть тела продлена до выходной границы расчетной области. На поверхности обтекаемого тела задано граничное условие для адиабатической стенки с логарифмическим законом для скорости и переменных турбулентности. На плоскостях симметрии задано граничное условие «симметрия». На входной и выходных границах области заданы неотражающие граничные условия.

При численном моделировании используется равномерная расчетная сетка $200 \times 100$ с разбиением до второго уровня в районе каверны (рис. 2). Разбиение ячеек до второго уровня означает последовательное деление каждой ячейки, попавшей в зону разбиения, пополам по каждому направлению. Размер ячейки в каверне составляет 0.3125 мм. Общее число ячеек составляет порядка 27000 . В основной части расчетов безразмерный шаг по времени был $0.03 \div 0.08$, расчеты с малыми характерными временами энерговыделения проводились с шагом $0.008 \div 0.016$.

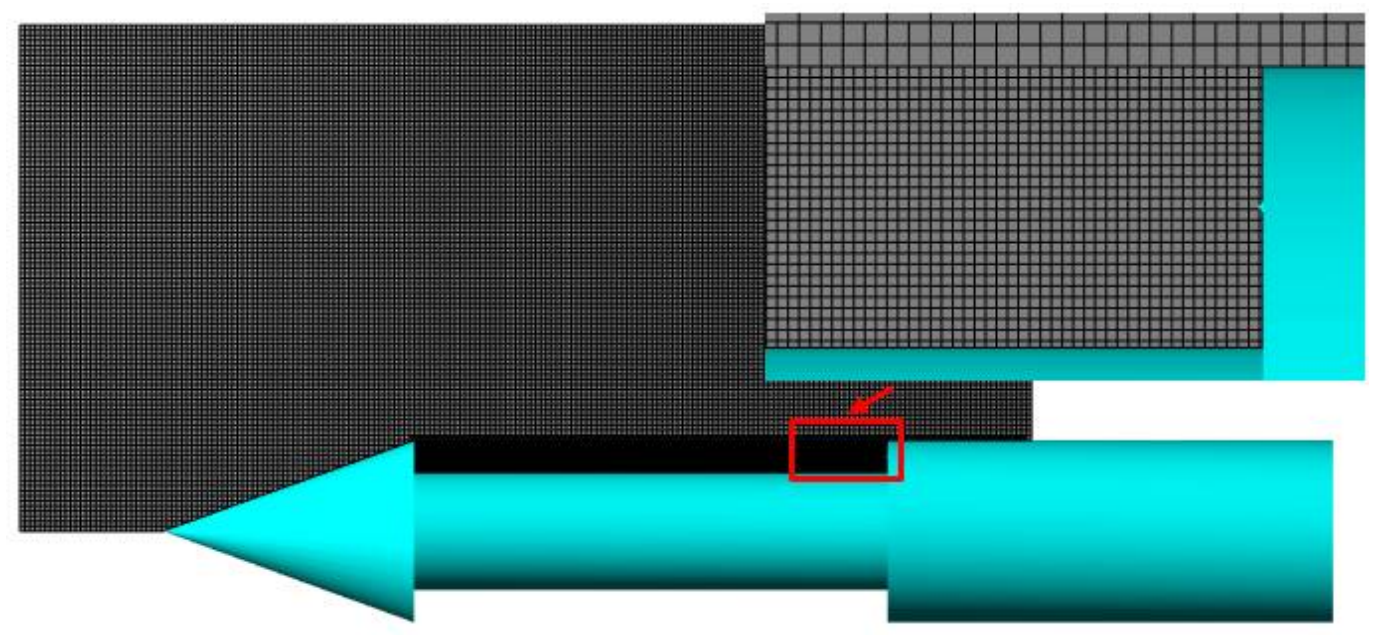

Рис. 2. Вид расчетной сетки

В качестве контрольного параметра использовался коэффициент профильного сопротивления модельного тела $c_{x}$ (без учета донного давления). В случае $L / h=10.8 c_{x}=0.524$ при замкнутой и $c_{x}=0.378$ при открытой схеме течения в каверне, соответственно.

\section{3. Результаты расчетов и их анализ}

Влияние положения источника постоянного теплового возмущения на перестройку структуры течения в каверне осуществлялось при мощности источника $N=0.104$. Время работы источника задавалось заведомо большим $-\tau=690$. Положение источника варьировалось в диапазоне $x=1.25 \div 6.25$. 
При расположении источника вне зоны влияния на переднюю отрывную область на расстоянии от переднего уступа каверны $x=4.375$ и больше открытие каверны не происходит (рис. $3, a$ ).Внесение возмущения приводит к отсоединению потока от дна каверны в месте внесения возмущения и к увеличению протяженности задней отрывной области вплоть до источника возмущения, при этом структура течения в окрестности передней отрывной области сохраняется неизменной. Во время работы источника коэффициент профильного сопротивления уменьшается (примерно на $12 \%$ при $x=4.375$ ) вследствие падения давления перед задним уступом каверны (рис. 4).После прекращения воздействия протяженность задней отрывной области сокращается, в каверне восстанавливается первоначальная замкнутая схема течения, профильное сопротивление возрастает до исходного значения.

$t=0$
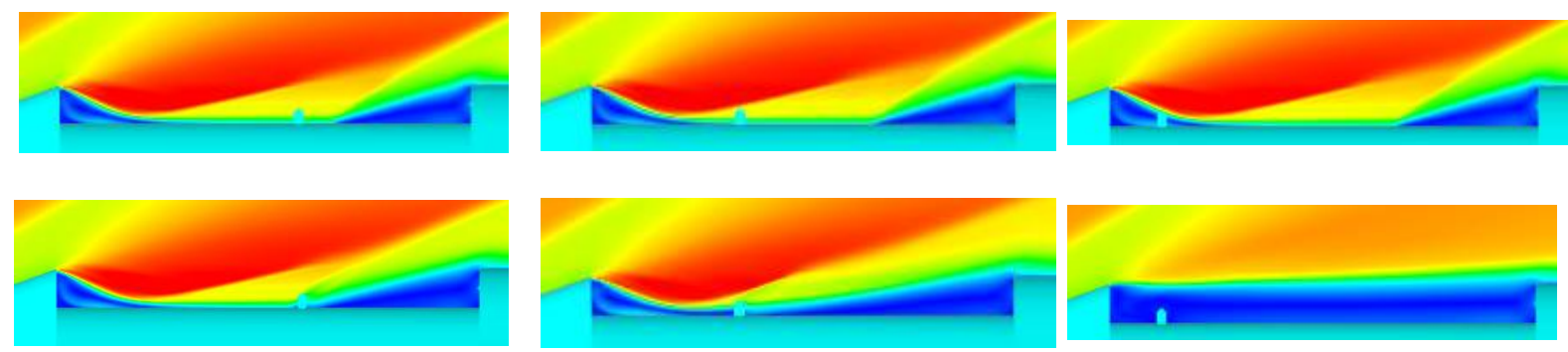

13
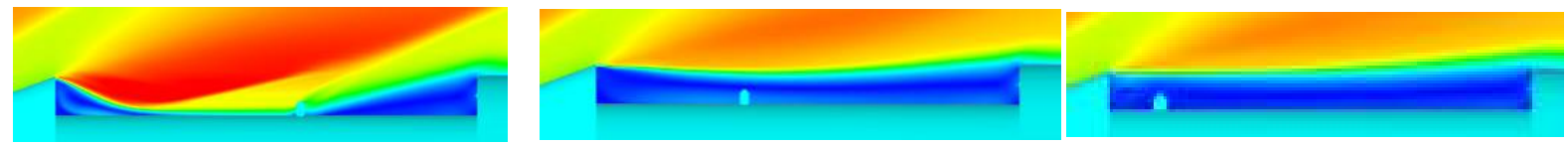

750

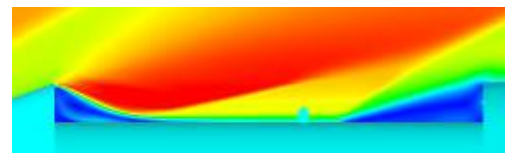

$a$

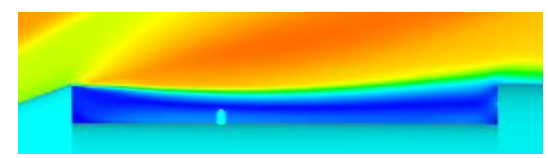

6

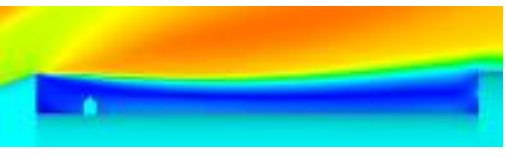

B

Рис. 3. Последовательные во времени (сверху вниз) поля числа Маха при $x=6.25(a) ; 3.75$ (б) и $1.25(6)$

При расположении источника на расстоянии от переднего уступа каверны $x=3.75$ и меньше происходит открытие каверны (рис. $3,6,6$ ).

В случае $x=3.75$ источник находится вне передней отрывной области (рис. $3, \sigma$ ). Как и в ранее рассмотренном случае $x \geq 4.375$, в начальные моменты времени происходит отсоединение потока от дна каверны в месте внесения возмущения и увеличение протяженности задней отрывной области вплоть до источника возмущения, однако при этом возмущение от источника проникает и в переднюю отрывную область. Вследствие повышения давления отрывная область за передним уступом расширяется вниз по потоку и объединяется с задней отрывной областью, образуя единую отрывную область. Во время энерговыделения коэффициент профильного сопротивления тела снижается до величины, несколько меньшей соответствующего значения при открытой каверне (рис. 4). После окончания воздействия устанавливается открытый режим обтекания каверны, коэффициент профильного сопротивления тела восстанавливается до величины, соответствующей значению при открытой каверне.

При $x=1.25$ источник энерговыделения находится внутри передней отрывной области (рис. 3, ) ). В этом случае включение источника приводит к повышению давления в передней отрывной области, вследствие чего происходит оттеснение слоя смешения передней отрывной области от дна каверны и распространение отрыва вплоть до объединения с задней отрывной областью. Как и в случае $x=3.75$, коэффициент профильного сопротивления снижается до величины, несколько меньшей соответствующего значения при открытой каверне, а 
после окончания воздействия при $t=690$ восстанавливается до величины, соответствующей значению при открытой каверне (рис. 4).

Следует отметить, что при расположении источника энерговыделения большой мощности вблизи переднего уступа ( $x \leq 3.75)$ открытие каверны в ряде случаев сопровождается пульсациями давления, при этом амплитуда колебаний коэффициента профильного сопротивления может достигать $\pm 5 \%$ от среднего значения. Для таких режимов на соответствующих графиках рис. 4 и далее приведены осредненные значения коэффициента профильного сопротивления.

Для рассмотренной мощности источника возмущений $N=0.104$ необратимая перестройка структуры течения в каверне происходит за достаточно короткий интервал времени, порядка $\Delta t=12$ в случае $x=3.75$ и $\Delta t=1.44$ в случае $x=1.25$. Последующее продолжающееся воздействие не приводит к структурным изменениям течения в каверне, среднее значение коэффициента профильного сопротивления практически не меняется до момента окончания воздействия при $t=690$ (рис. 4). По окончании энерговыделения происходит падение давления в области между источником и передним уступом с последующим его восстановлением до величины, соответствующей течению в открытой каверне, что отражается на графиках изменения коэффициента профильного сопротивления (рис. 4)

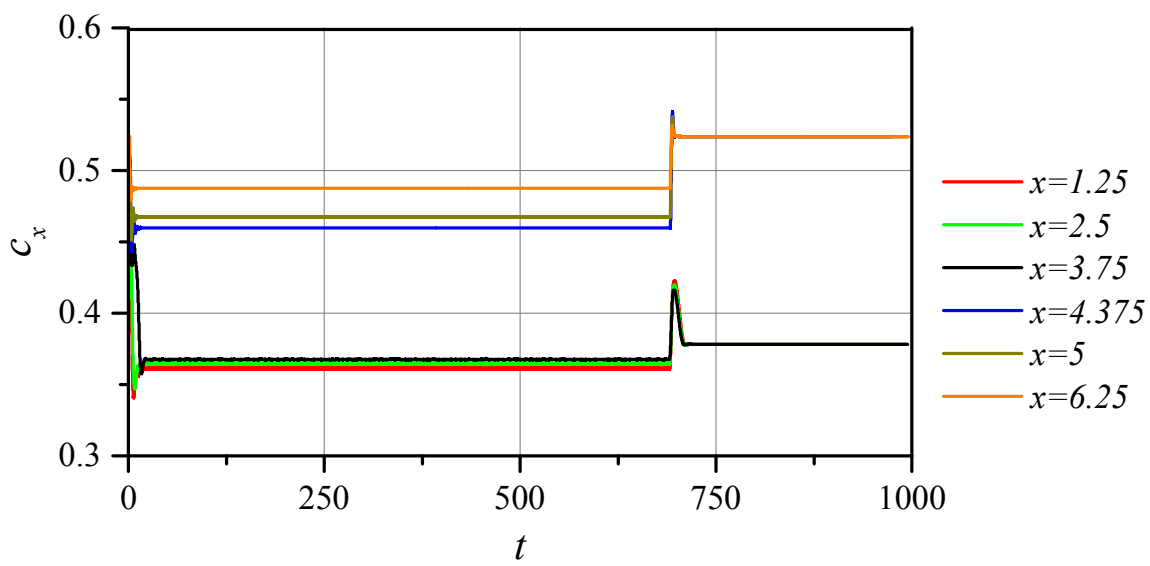

Рис. 4. Изменение коэффициента профильного сопротивления тела при различных значениях расстояния $x$ от переднего уступа до источника возмущения

Таким образом, в области гистерезиса локальный энергоподвод может привести к необратимой перестройке первоначально замкнутой каверны в открытую каверну и снижению профильного сопротивления тела. Положение источника энергоподвода существенно влияет на открытие каверны. Для рассмотренной мощности $N=0.104$ и достаточно большом времени его работы необратимое открытие каверны происходит при $x \leq 3.75$.

С целью оценки пороговой мощности, необходимой для открытия каверны, была проведена серия расчетов при фиксированной мощности источника энерговыделения для случаев $x=1.25,2.5$ и 3.75. При $x=1.25$ источник находится внутри передней отрывной области около переднего уступа, при $x=2.5$ - на границе передней отрывной области и при $x=3.75$ - вне этой области. Длительность работы источника задавалась заведомо большой. Расчеты показали, что при удалении источника возмущений от переднего уступа каверны пороговая мощность возрастает. Так, при $x=1.25$ открытие каверны происходит при мощности большей или равной $N=0.0311$ (рис. 5), при $x=2.5-N=0.0415$, а при $x=3.75-N=0.083$.

Важной характеристикой энергетического воздействия, способного вызвать необратимую перестройку структуры течения в каверне, является величина затраченной энергии. Расчеты показывают, что при увеличении мощности источника энергоподвода выше установленного порогового значения уменьшается время, необходимое для перестройки течения в каверне к структуре, предшествующей необратимому открытию каверны (рис. 5). В случае 
$x=1.25$ при пороговом значении $N=0.0311$ характерное время работы источника, необходимое для необратимого открытия каверны, достигает $\tau=9.7$, в случае $N=0.0415 \tau=4.49$, а при $N=0.104 \tau=1.45$. При этом затрачиваемая на открытие каверны энергия $N_{\tau}$ снижается с $N_{\tau}=0.301$ до 0.186 и 0.15 , соответственно. Аналогичная тенденция прослеживается при других расположениях источника.

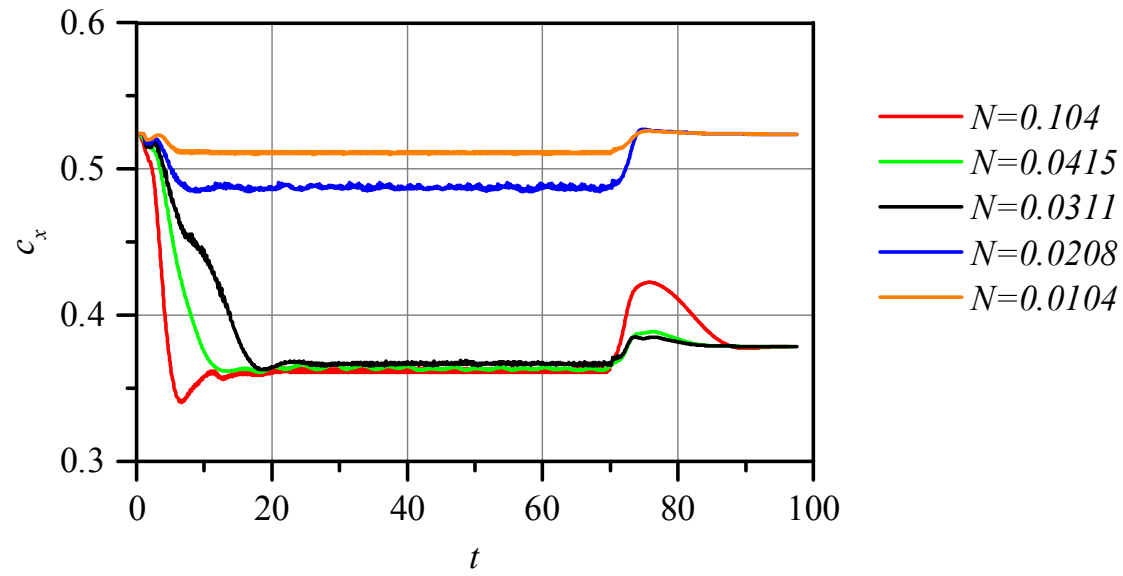

Рис. 5. Изменение во времени коэффициента сопротивления при $x=1.25$ и различной мощности источника энергоподвода

При расположении источника энергоподвода на расстоянии $x=3.75$ от переднего уступа замкнутой каверны и мощности источника $N=0.104$ характерное время работы источника, необходимое для необратимого открытия каверны, достигает $\tau=12.4$. Соответственно, при $x=2.5$ и $N=0.052$ характерное время достигает $\tau=6.2$, а при $x=1.25$ и $N=0.0415-\tau=4.49$. Величина энергии, выделяемой источником за указанное характерное время, составляет $N_{\tau}=1.29,0.32$ и 0.186 для $x=3.75,2.5$ и 1.25 , соответственно. Таким образом, затраты энергии, необходимой для необратимой перестройки течения в каверне, при расположении источника энерговыделения внутри передней отрывной области $(x<2.5)$ снижаются в несколько раз по сравнению со случаем расположения источника за пределами этой области.

Рассматривался вариант подвижного источника постоянной мощности, движущегося от заднего уступа каверны к переднему с постоянной скоростью. Скорость движения источника такова, что он проходит всю каверну за время $t=596.5$. Начальное положение источника было $x=6.25$. Во время движения источник работал непрерывно. Мощность источника варьировалась в диапазоне $N=0.0208 \div 0.519$. Расчеты показали, что при малой величине мощности $N=0.0208$ перестройка течения в каверне не происходит (рис.6). Чем больше мощность источника, тем на более удаленном расстоянии источника от переднего уступа каверны происходит необратимая перестройка течения. При мощности $N=0.0311$ необратимая перестройка течения в каверне происходит при положении источника на расстоянии $x=2$. При $x>3$ необходимая пороговая мощность существенно возрастает, достигая при $x=5$ значения порядка $N \sim 0.5$.

Второй вариант изменяющегося источника - неподвижный источник, мощность которого линейно растет со временем. В данной задаче варьируется положение источника возмущения $x$. Во всех случаях мощность источника увеличивается по закону: $N=0.000156 t$. Из результатов решения видно, что для того, чтобы вызвать необратимую перестройку течения с помощью источника, расположенного на расстоянии $x=0.625 \div 3.125$, необходима мощность $N=0.03 \div 0.05$, в то время как для источника, расположенного на расстоянии $x=3.75$ от переднего уступа необходима мощность $N \sim 0.1$ (рис. 7). 


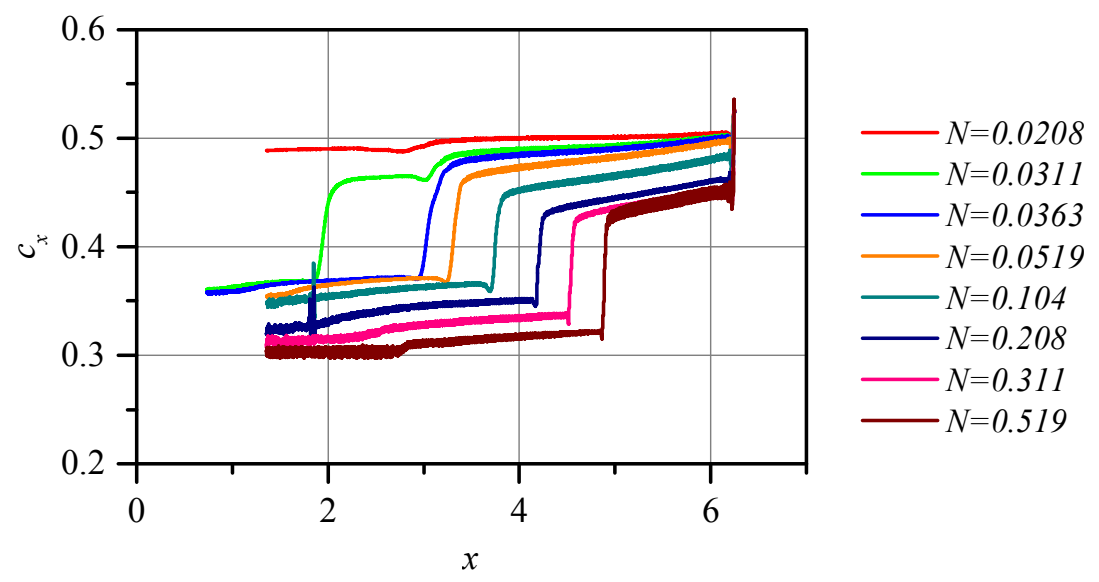

Рис. 6. Изменение коэффициента сопротивления в зависимости от положения источника энерговыделения при его перемещении вдоль каверны для различных значений мощности источника

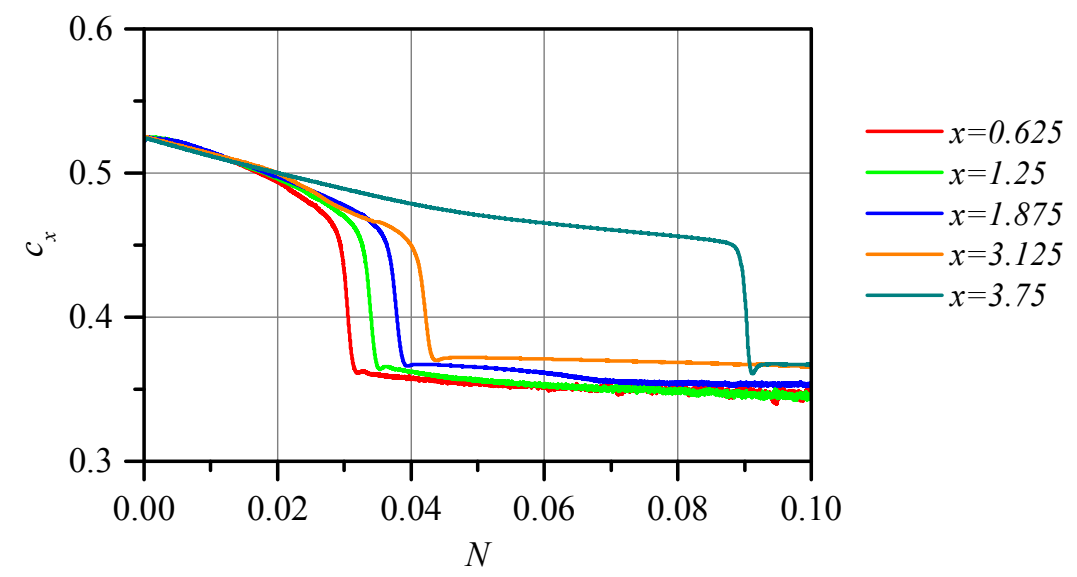

Рис. 7. Изменение коэффициента сопротивления в зависимости от мощности источника при различных положениях источника

На основе полученных результатов получена зависимость пороговой мощности $N$, необходимой для необратимой перестройки течения, от положения х источника возмущений (рис. 8). При $x<3$ пороговая мощность $N$ слабо зависит от $x$ и изменяется в диапазоне от 0.03 до 0.045 . При $x>3$ пороговая мощность источника возмущений существенно возрастает по мере увеличения $x$.

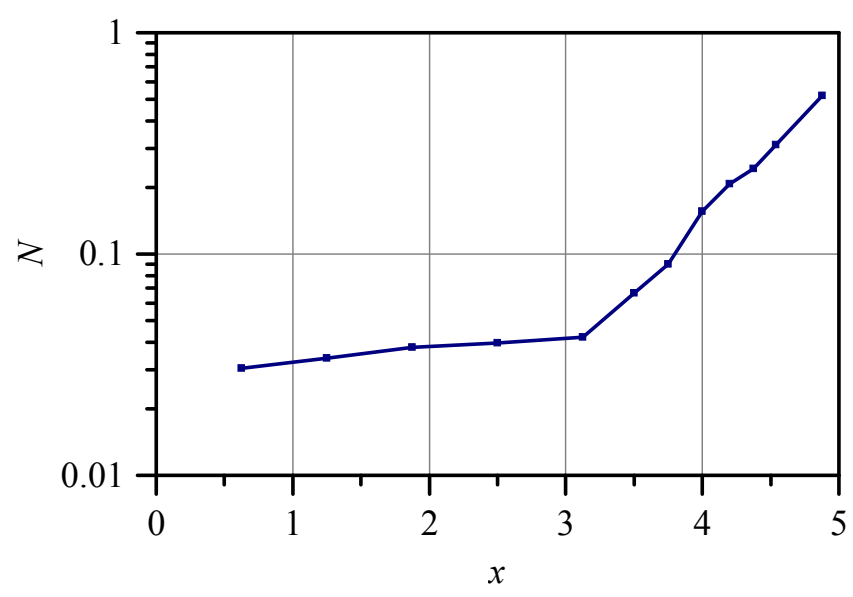

Рис. 8. Зависимость пороговой мощности источника от расстояния до переднего уступа каверны 


\section{4. Заключение}

На примере численного решения задачи о сверхзвуковом обтекании кольцевой каверны с расположенным в ней источником внешнего энергоподвода исследована восприимчивость отрывных структур около каверны к газодинамическим возмущениям, порождаемым источником в зависимости от параметров его локализации, интенсивности и длительности действия. Для режимов гистерезисного обтекания показано, что маломощный кратковременный тепловой импульс способен вызывать необратимую перестройку от замкнутой к открытой схеме отрывного обтекания каверны с сопутствующим скачкообразным снижением ее аэродинамического сопротивления. Получены оценки минимальных затрат энергии, необходимых для данной перестройки.

\section{Благодарности и ссылки на гранты}

Работа выполнена при частичной финансовой поддержке фонда Президента Российской Федерации (грант МК-5514.2016.1) и РФФИ (грант 19-01-00242).

\section{Литература}

1. White E.B., Ergin F.G. Receptivity and Transient Growth of Roughness-Induced Disturbances // AIAA. 2003-4243. Pp. 1-11.

2. Hamed A., Das K., Basu D. Numerical Simulations of Fluidic Control for Transonic Cavity Flows // 42-ndAIAA Aerospace Sciences Meeting \& Exhibit: Book of abstracts. - January 5-8, 2004.

3. Знаменская И.А., Латфуллин Д.Ф., Мурсенкова И.В. Ламинарно-турбулентный переход в сверхзвуковом пограничном слое при инициировании импульсного поверхностного разряда // Письма в "Журнал технической физики". 2008. Т. 34, № 15. С. 75-80.

4. Аульченко С.М., Замураев В.П., Знаменская И.А., и др. О возможности управления трансзвуковым обтеканием профилей с помощью подвода энергии на основе наносекундного разряда типа “плазменный лист"// Журнал технической физики. 2009. Т.79. №3. С. 17-27.

5. Аксенов В.С., Голуб В.В., С.А. Губин, и др. Сверхзвуковое обтекание воздухом профиля крыла при инициировании скользящего разряда на его поверхности // Плазменная аэродинамика в сверхзвуковом потоке газа. 2010. № 1 (дополнительный). С. 93-101.

6. Георгиевский П.Ю., Левин В.А. Сверхзвуковое обтекание тел при наличии внешних источников тепловыделения // Письма в "Журнал технической физики". 1988. Т. 14. № 8. С. 684.

7. Георгиевский П.Ю., Левин В.А. Управление обтеканием различных тел с помощью локализованного подвода энергии в сверхзвуковой набегающий поток // Изв. РАН. МЖГ. 2003. № 5. C. $154-167$.

8. Третьяков П.К., Гаранин А.Ф., Грачев Г.Н. и др. Управление сверхзвуковым обтеканием тел с использованием мощного оптического пульсирующего разряда // Доклады РАН. 1996. Т. 351. № 3. C. 339-340.

9. Гувернюк С.В., Самойлов А.Б. Об управлении сверхзвуковым обтеканием тел с помощью пульсирующего теплового источника // Письма в "Журнал технической физики". 1997. Т. 23, № 9. C. $1-8$.

10. Георгиевский П.Ю., Левин В.А. Режимы сверхзвукового обтекания тел в условиях энерговклада различной геометрической конфигурации в набегающий поток // Теплофизика высоких температур. 2010. Т. 48, № 1 (приложение). С. 77-84.

11. Guvernyuk S.V. Comparison of energetic and dynamic devices of non-uniformity formation in the supersonic flow around a blunt body / Proc. of the 3rd Workshop on Magneto-Plasma Aerodynamics in Aerospace Applications. - Moscow. IVTAN. 2001. Pp. 226-231. https://istina.msu.ru/publications/article/8306359/ 
12. Гувернюк С.В., Синявин А.А. Об управлении гистерезисом сверхзвукового обтекания прямоугольной каверны с помощью теплового импульса / Успехи механики сплошных сред: к 70летию академика В.А. Левина: сб. науч. тр. - Владивосток: Дальнаука, 2009. С. 196-203. https://istina.msu.ru/publications/article/214241/

13. Калугин А.И., Луценко А.Ю., Столярова Е.Г. Гистерезисные явления при обтекании затупленных тел в трансзвуковом потоке // Космонавтика и ракетостроение. 2009. Вып. 1 (54). С. 79-93.

14. Гужавин А.И., Коробов Я.П. О гистерезисе сверхзвуковых отрывных течений // Изв. АН СССР. МЖГ. 1984. №2. С. 116-125.

15. Charwat A.F., Roos J.N., Dewey F.C., Hitz J.A. An investigation of separated flows. Part I: The pressure field // J. Aerosp. Sci. 1961. V. 28, № 6, Pp. 457-470. https://doi.org/10.2514/8.9037

16. Charwat A.F., Roos J.N., Dewey F.C., Hitz J.A. An investigation of separated flows. Part II: Flow in the cavity and heat transfer//J. Aerosp. Sci. 1961. V.28, №6, Pp.513-527. https://doi.org/10.2514/8.9099

17. Stalling R. L., Wilcox F. J. Experimental Cavity Pressure Distribution at Supersonic Speeds // NASA TP 2683. 1987.

18. Zhang J., Morishita E., Okunuki T., Itoh H. Experimental investigation on the mechanism of flow-type changes in supersonic cavity flows // Trans. Jpn. Soc. Aeronaut. Space Sci. 2002. V.45, № 149, pp. 170-179. https://doi.org/10.2322/tjsass.45.170

19. Чжен П. Отрывные течения. Том II. М.: Мир, 1973. 280 с.

20. Швец А.И. Исследование течения в цилиндрическом вырезе на осесимметричном теле при сверхзвуковом обтекании // Изв. РАН. МЖГ. 2002. №1. С. 123-131.

21. Гувернюк С.В., Зубков А.Ф., Симоненко М.М., Швец А.И. Экспериментальное исследование трехмерного сверхзвукового обтекания осесимметричного тела с кольцевой каверной // Изв. РАН. МЖГ. 2014. № 4. С. 136-142.DOI: 10.1134/S0015462814040140

22. Гувернюк С.В., Зубков А.Ф., Симоненко М.М. Экспериментальное исследование сверхзвукового обтекания осесимметричной кольцевой каверны // Инженерно-физический журнал. 2016. T. 89. № 3. C. 670-679. DOI: 10.1007/s10891-016-1426-4

23. Гувернюк С.В., Зубков А.Ф., Симоненко М.М. О сверхзвуковом обтекании кольцевых каверн под углом атаки // Физико-химическая кинетика в газовой динамике. 2018. Т. 19. Вып. 1. http://chemphys.edu.ru/issues/2018-19-1/articles/734/

24. Mohri K., Hillier R. Computational and experimental study of supersonic flow over axisymmetric cavities // Shock Waves. 2011. Vol. 21. P. 175-191. https://doi.org/10.1007/s00193-011-0312-4

25. Иванов И.Э., Крюков И.А., Ларина Е.В., Тарасевич А.Г. Численное моделирование обтекания осесимметричного тела с кольцевой каверной // Физико-химическая кинетика в газовой динамике. 2015. Т. 16. Вып. 2. С.1-10. http://chemphys.edu.ru/issues/2015-16-2/articles/583/

26. Ivanov I.E., Kryukov I.A., Larina E.V., Glushko G.S. Turbulent flow over an axisymmetric body with annular cavity // Journal of Physics: Conference Series. 2017. V. 815. № 1. Pp. 1-8.

27. Шишаева А.С., Симоненко М.М., Гувернюк С.В., Аксенов А.А. Численное моделирование аэродинамического гистерезиса при сверхзвуковом обтекании осесимметричного тела с каверной в программном комплексе FlowVision // Физико-химическая кинетика в газовой динамике. 2017. Т. 18, вып. 1.http://chemphys.edu.ru/issues/2017-18-1/articles/696/

28. Жлуктов С.В., Аксенов А.А., Харченко С.А., и др. Моделирование отрывных течений в программном комплексе FlowVision-HРC//Вычислительные методы и программирование, 2010, T. 11, № 2, C. 76-87. http://num-meth.srcc.msu.ru/zhurnal/tom_2010/pdf/v11r128.pdf

29. Aksenov A., Dyadkin A., Pokhilko V.Overcoming of barrier between CAD and CFD by modified finite volume method// ASME-PUBLICATIONS-PVP, 1998. V.337, Pp.79-83.

30. Markova T.V., Aksenov A.A., Zhluktov S.V., Savitsky D.V., Gavrilov A.D., Son E.E., Prokhorov A.N. Simulating flow around scaled model of a hypersonic vehicle in wind tunnel // Journal of Physics: Conference Series. 2016. V. 774. № 1. Pp. 1-9. https://iopscience.iop.org/article/10.1088/1742$\underline{6596 / 774 / 1 / 012095 / p d f}$ 


\section{References}

1. White E.B., Ergin F.G. Receptivity and Transient Growth of Roughness-Induced Disturbances. AIAA. 2003-4243. pp. 1-11.

2. Hamed A., Das K., Basu D. Numerical Simulations of Fluidic Control for Transonic Cavity Flows. 42nd AIAA Aerospace Sciences Meeting \& Exhibit: Book of abstracts, January 5-8, 2004.

3. Znamenskaya I. A., Latfullin D. F., Mursenkova I. V. Pis'ma v "Zhurnaltekhnicheskoifiziki", 2008, V. 34, № 15, pp. 75-80.

4. AulchenkoS. M., ZamuraevV. P., ZnamenskayaI. A., KalininaA. P., OrlovD. M., SisouevN. N. Zhurnal tekhnicheskoi fiziki, 2009, V. 79, № 3, pp. 17-27.

5. Aksenov V.S., Golub V.V., Gubin S.A., Savel'ev A. S., Sechenov V. A., Son E. E. Plazmennaya aerodinamika v sverkhzvukovom potoke gaza, 2010, № 1 (dopolnitel'nyi), pp. 93-101.

6. GeorgievskiiP.Yu., Levin V.A. Pis'ma v "Zhurnaltekhnicheskoifiziki", 1988, V. 14, No 8, p. 684.

7. GeorgievskiiP.Yu., Levin V.A. Izv. RAN. MZhG, 2003, № 5, pp. 154-167.

8. Tret'yakov P.K., Garanin A.F., Grachev G.N., Krainev V.L., Ponomarenko A.G., Ivanchenko A.I., Yakovlev V.I. Doklady RAN. 1996. V. 351. № 3. pp. 339-340.

9. Guvernyuk S. V., Samoilov A. B. Pis'ma v "Zhurnal tekhnicheskoi fiziki". 1997. V. 23, № 9. pp. 1-8.

10. GeorgievskiiP.Yu., LevinV.A. Teplofizika vysokikh temperatur. 2010. V. 48, № 1 (prilozhenie). pp. $77-84$.

11. Guvernyuk S.V. Comparison of energetic and dynamic devices of non-uniformity formation in the supersonic flow around a blunt body, Proc. of the 3rd Workshop on Magneto-Plasma Aerodynamics in Aerospace Applications., Moscow, IVTAN, 2001, pp. 226-231.

12. Guvernyuk S.V., Sinyavin A.A. Uspekhi mekhaniki sploshnykh sred: k 70-letiyu akademika V.A. Levina: sb. nauch. tr. Vladivostok, Dal'nauka, 2009, pp. 196-203.

13. Kalugin A.I., LutsenkoA.Yu., Stolyarova E.G. Kosmonavtika I raketostroenie, 2009, V. 1, pp. 79-93.

14. Guzhavin A.I., KorobovYa.P. Izv. AN SSSR. MZhG, 1984, №2. pp. 116-125.

15. CharwatA.F., RoosJ.N., DeweyF.C., HitzJ.A. An investigation of separated flows. Part I: The pressure field, J. Aerosp. Sci, 1961. V. 28, № 6, pp. 457-470.

16. Charwat A.F., Roos J.N., Dewey F.C., Hitz J.A.An investigation of separated flows. Part II: Flow in the cavity and heat transfer,J. Aerosp. Sci, 1961, V. 28, № 6, pp. 513-527.

17. Stalling R. L., Wilcox F. J.Experimental Cavity Pressure Distribution at Supersonic Speeds, NASA TP 2683, 1987.

18. Zhang J., Morishita E., Okunuki T.,Itoh H. Experimental investigation on the mechanism of flow-type changes in supersonic cavity flows, Trans. Jpn. Soc. Aeronaut. Space Sci, 2002, V. 45, № 149, pp. 170 179 .

19. Chzhen P. Otryvnyetecheniya (Separated flows). Vol. II. Moscow: Mir, 1973. 280 p.

20. Shvets A.I. Izv. RAN. MZhG, 2002, №1. pp. 123-131.

21. Guvernyuk S.V., Zubkov A.F., Simonenko M.M., Shvets A.I. Izv. RAN. MZhG, 2014, № 4, pp. 136142.

22. GuvernyukS. V., ZubkovA. F., SimonenkoM. M. Inzhenerno-fizicheskiizhurnal, 2016, V. 89, № 3, pp. 670-679.

23. GuvernyukS. V., ZubkovA. F., SimonenkoM. M. Fiziko-khimicheskaya kinetika v gazovoi dinamike, 2018, V. 19, Iss. 1, available at: http://chemphys.edu.ru/issues/2018-19-1/articles/734/

24. Mohri K., Hillier R. Computational and experimental study of supersonic flow over axisymmetric cavities,Shock Waves, 2011, Vol. 21, pp. 175-191.

25. IvanovI.E., KryukovI.A., LarinaE.V, Tarasevich A.G. Fiziko-khimicheskaya kinetika v gazovoi dinamike, 2015, V. 16, Iss. 2, pp. 1-10, available at: http://chemphys.edu.ru/issues/2015-16-2/articles/583/

26. IvanovI.E., KryukovI.A., LarinaE.V., GlushkoG.S. Turbulent flow over an axisymmetric body with annular cavity Journal of Physics: Conference Series, 2017, V. 815, № 1, pp. 1-8. 
27. Shishaeva A. S., Simonenko M. M., Guvernyuk S. V., Aksenov A. A. Fiziko-khimicheskaya kinetika $v$ gazovoi dinamike, 2017, V. 18, Iss. 1, available at: http://chemphys.edu.ru/issues/2017-18-1/articles/696/

28. ZhluktovS.V., AksenovA.A., KharchenkoS.A., MoskalevI. V., SushkoG. B., ShishaevaA. S. Vychislitel'nye metody $i$ programmirovanie, 2010, V.11, N2, pp.76-87, available at: http://nummeth.srcc.msu.ru/zhurnal/tom 2010/pdf/v11r128.pdf

29. Aksenov A., Dyadkin A., Pokhilko V. Overcoming of barrier between CAD and CFD by modified finite volume method, ASME-PUBLICATIONS-PVP, 199, V. 337, pp.79-83.

30. Markova T.V., Aksenov A.A., Zhluktov S.V., Savitsky D.V., Gavrilov A.D., Son E.E., Prokhorov A.N. Simulating flow around scaled model of a hypersonic vehicle in wind tunnel. Journal of Physics: Conference Series. 2016, V. 774, № 1, pp. 1-9, available at: https://iopscience.iop.org/article/10.1088/1742-6596/774/1/012095/pdf

Статья поступила в редакцию 19 октября 2019 г. 